\title{
Controlling the dual cascade of two-dimensional turbulence
}

\author{
M. M. F A R A Z MA N D ${ }^{1}$, N.K. - R. K E V L A HA $\mathrm{N}^{2}$ \\ AND B. PROTAS ${ }^{2}$ \\ ${ }^{1}$ School of Computational Engineering \& Science, \\ ${ }^{2}$ Department of Mathematics \& Statistics, \\ McMaster University, Hamilton L8S 4K1, Canada
}

(Received September 1, 2010)

The Kraichnan-Leith-Batchelor (KLB) theory of statistically stationary forced homogeneous isotropic 2-D turbulence predicts the existence of two inertial ranges: an energy inertial range with an energy spectrum scaling of $k^{-5 / 3}$, and an enstrophy inertial range with an energy spectrum scaling of $k^{-3}$. However, unlike the analogous Kolmogorov theory for 3-D turbulence, the scaling of the enstrophy range in 2-D turbulence seems to be Reynolds number dependent: numerical simulations have shown that as Reynolds number tends to infinity the enstrophy range of the energy spectrum converges to the KLB prediction, i.e. $E \sim k^{-3}$. The present paper uses a novel optimal control approach to find a forcing that does produce the KLB scaling of the energy spectrum in a moderate Reynolds number flow. We show that the time-space structure of the forcing can significantly alter the scaling of the energy spectrum over inertial ranges. A careful analysis of the optimal forcing suggests that it is unlikely to be realized in nature, or by a simple numerical model.

\section{Introduction}

In 1941 Kolmogorov proposed a statistical theory for homogeneous, isotropic and statistically stationary three-dimensional incompressible turbulence (Kolmogorov 1941). He assumed that there is an inertial range of length scales in which the effect of the external forcing and the molecular viscosity are negligible. Since in the inertial range the energy spectrum, $E(k)$, depends only on the mean energy dissipation rate $\epsilon$ and the wavenumber $k$, dimensional analysis shows that the energy spectrum must follow the universal form:

$$
E(k)=C \epsilon^{2 / 3} k^{-5 / 3}
$$

where $C$ is a universal constant. Moreover, he conjectured that in three dimensions the turbulent energy is transferred from larger scales (lower wavenumbers) to smaller scales (higher wavenumbers) where the energy is eventually dissipated by viscosity. The $k^{-5 / 3}$ prediction has been verified to high accuracy in numerous experiments and computations (apart from small corrections due to the intermittency of the energy dissipation rate).

In spite of the fact that there are no truly two-dimensional flows in nature, many flows may be well-described by two-dimensional models in the appropriate limit. For example, certain properties of atmosphere and ocean flows can be explained by 2-D Navier-Stokes equations (Frisch 1995; Lindborg 1999). Such applications, in addition to the fact that the 2-D Navier-Stokes equations are more mathematically tractable, has led to many studies of two-dimensional flows over the last few decades. The success and simplicity of Kolmogorov's theory has inspired efforts to adapt the theory to two-dimensional turbulence. 
However, Kolmogorov's theory does not apply directly to two-dimensional flow since the dynamics of two-dimensional flows are qualitatively different from three-dimensional flows. For example, vortex stretching which plays a key role in energy transfer between scales in 3-D is absent in 2-D. In addition, Fjørtoft (1953) (and later Merilees \& Warn (1975) and Gkioulekas \& Tung (2007)) showed that in a 2-D incompressible NavierStokes flow the energy is (on average) transferred to larger scales, while the enstrophy is transferred to smaller scales. This so-called dual cascade is quite different from the 3-D case where the energy cascades down to smaller scales in the inertial range. Based on Fjørtoft's work and Kolmogorov's universality assumption, Kraichnan (1967), Leith (1968) and Batchelor (1969) developed an analogous theory (usually referred to as KLB theory) for homogeneous, isotropic and statistically stationary two-dimensional forced turbulence.

According to the KLB theory, in 2-D Navier-Stokes turbulence, there are two inertial ranges (of energy and enstrophy respectively) where the effects of the viscosity and the external forces are negligible. The energy and the enstrophy are injected by external forcing in some intermediate scales between energy and enstrophy inertial ranges. The injected energy is then transferred to ever larger scales through the energy inertial range while the enstrophy is transferred to smaller scales through the enstrophy inertial range until it is eventually dissipated by molecular viscosity. Kraichnan assumed that in the energy inertial range the energy spectrum of the flow, $E(k)$, depends only on the energy dissipation rate $(\epsilon)$ and the wavenumber $k$, while, in the enstrophy inertial range, $E(k)$ depends only on enstrophy dissipation rate $(\eta)$ and $k$. Using dimensional analysis, he then predicted the following scaling laws: $E(k) \propto k^{-5 / 3}$ in the energy inertial range (just as in three-dimensional turbulence) and $E(k) \propto k^{-3}$ (with a possible logarithmic correction (see Kraichnan 1971)) in the enstrophy inertial range (see figure 1).

Many numerical and laboratory experiments have been performed in attempts to test the KLB theory (see, for instance, Lilly 1969; Borue 1993; Pasquero \& Falkovich 2002; Boffetta 2007; Sommeria 1986; Marteau et al. 1995; Bruneau \& Kellay 2005, also see Clercx \& van Heijst (2009) for a recent review.). These experiments confirm the general setting of the theory. Each of the cascades have been observed independently with the predicted slopes. However, there is a controversy. KLB theory predicts that if enough energy and enstrophy are injected into the system these dual cascades (i.e. inverse cascade of energy and forward cascade of enstrophy) must be realizable simultaneously in a statistically stationary state. (Indeed, the inverse cascade of energy can be only quasistationary in an infinite domain since the energy is transferred to ever larger scales.) In the numerical and experimental studies, which attempt to realize the dual cascades of 2-D turbulence simultaneously, the $-5 / 3$ slope of the inverse cascade has been well established (see Smith \& Yakhot 1993; Frisch \& Sulem 1984, also see Scott (2007) for a slightly steeper spectrum in the quasi-stationary state). However, slopes significantly steeper than -3 have been usually reported for the forward cascade. Recently, some very high resolution numerical simulations have been able to achieve scalings of the enstrophy cascade close to $k^{-3}$. To the best of our knowledge, Boffetta \& Musacchio (2010) has achieved the result closest to the KLB prediction. At the highest Reynolds number (i.e. at a resolution of $32768^{2}$ ) their enstrophy cascade has a slope of approximately -3.35 . They present persuasive evidence that the enstrophy range approaches the KLB scaling asymptotically in the limit of infinite Reynolds number (also see Boffetta (2007) and Bracco \& McWilliams (2010) for similar studies). While scaling of the energy cascade is not Reynolds number dependent, the source of the Reynolds number dependence of the enstrophy range remains poorly understood.

Some attempts have been made to explain this departure from the KLB theory. First, 
it should be noted that while the KLB theory assumes unbounded domains the numerical and laboratory experiments are necessarily performed on bounded domains. Kraichnan (1967) pointed out from the very beginning that this may affect the results of the experiments since the energy transferred by the inverse cascade accumulates in the largest available scales. This problem is partially avoided by adding a friction-type dissipation to remove energy at the largest scales. This type of dissipation, usually called Rayleigh (or Ekman) damping, resembles the friction between the atmospheric flow and the earth's surface (Basdevant et al. 1981). On the other hand, Tran \& Dritschel (2006) (also see Tran et al. 2007) disproved one of the underlying assumption of the KLB theory: that enstrophy dissipation converges to a non-zero value in the zero molecular viscosity limit. (This prediction is the analogue of the prediction that energy dissipation rate converges to a non-zero finite value as Reynolds number tends to infinity in three-dimensional turbulence.) However, Tran et al. (2007) showed (by substituting enstrophy dissipation with a Reynolds number dependent quantity) that the -3 slope of the enstrophy cascade should still hold.

Another question which is still not well understood is the effect of the forcing on the dual cascades in forced-dissipative two-dimensional turbulence. Studies have shown that the type of forcing modifies the slope of the enstrophy inertial range spectrum. The convention is that a monoscale or a monoscale-like (band-limited) forcing is used. The forcing is confined to a single wave number in the case of monoscale forcing, or to a few adjacent wavenumbers in the band-limited case. The input energy is transferred to larger scales and the input enstrophy to the smaller scales as predicted by KLB. This type of forcing, first suggested by Kraichnan (1967), has some advantages. First, it is easy to control the rate of energy and enstrophy injection and secondly, the energy and enstrophy injection ranges do not overlap with the inertial ranges. It is therefore consistent with the inertial range hypothesis that conjectures that the energy input is negligible in the inertial ranges.

However, in 1994 Constantin et al. (1994) proved that, in a finite domain, monoscale forcing cannot produce dual cascades with the slopes predicted by KLB. Later, Tran \& Shepherd (2002) and Tran \& Bowman (2003) generalized this result to band-limited forcing and more general types of dissipation. They proved that with monoscale (or band-limited) forcing, the slope of the energy spectrum in the forward cascade cannot be shallower than -5 . These results show that monoscale and band-limited forcing are actually inconsistent with KLB theory. Tran \& Shepherd (2002) show that in the presence of "inverse viscosity" (which removes energy from large scales) the KLB scaling is theoretically possible. However, as was mentioned before, this result suffers from the lack of numerical and experimental evidence.

On the other hand, Lundgren (2003) proposed a linear forcing (directly proportional to the velocity) to study three-dimensional turbulence. Lundgren was motivated by the need to find a forcing that has a better physical justification and is easier to apply in non-spectral simulations than the usual spectral forcing applied only to a few small wavenumbers. Later, Rosales \& Meneveau (2005) further studied the effects of this type of forcing. Their results show that the predictions of the Kolmogorov theory still hold under linear forcing, despite the fact that linear forcing is active at all scales.

In two dimensions, the effects of linear damping which is active in all scales (as opposed to Rayleigh friction which is active only at the largest scales) has been studied in recent years (Boffetta et al. 2002; Tsang et al. 2005; Tsang \& Young 2009). It should be noted that both linear forcing and linear damping are active over all scales, including the energy and enstrophy inertial ranges. This appears to violate the inertial range hypothesis because energy is injected (removed) directly at (from) all scales. However, the above 
mentioned studies show that the energy flux (and enstrophy flux in 2-D case) remains almost constant over a wide range of wavenumbers, even when linear forcing or damping is applied. These observations raise the question of whether there exist types of forcing (possibly active at all scales) which are able to produce the dual cascades with the scaling laws predicted by KLB theory. Answering this question is one of the goals of the present paper.

The other goal is to investigate the effect of the space-time structure of the bandlimited forcing on the energy spectrum. In most previous numerical simulations, the forcing is random in phase (see e.g. Schorghofer 2000). In some simulations, the forcing is delta-correlated in time (see e.g. Boffetta 2007). In some others, the time correlation is increased by a Markov process (see e.g. Lilly 1969). There are also simulations in which the forcing depends on the instantaneous velocity field (see e.g. Chen et al. 2003). The motivation for using each of these forcings is to have a control on the energy and enstrophy injection rates. Therefore, there is no unique and physically well-justified way to define the forcing. Almost always, the effect of the space-time structure of the forcing on the energy spectrum is neglected. Here, we show that the details of this space-time structure can have determining effects on the slopes of the cascades even when the forcing is band-limited.

Next sections are organized as follows. In $\S 2$, we give a brief introduction to adjointbased optimal control and introduce our method for controlling the energy spectrum of a flow. The numerical results of the application of the control process to turbulent flows are presented and discussed in $\S 3$. These results are divided into two parts. In the first part full-band forcing is considered (§3.1), while in the second part the forcing is band-limited (§3.2). Finally, in $\S 4$ we explore the significance of our results for the theory of two-dimensional turbulence and suggest further applications of our optimal control approach.

\section{Problem Formulation}

Consider the incompressible Navier-Stokes equations on a two-dimensional box $\mathbb{T}^{2}$ with doubly periodic boundary conditions.

$$
\begin{gathered}
\mathcal{L} \mathbf{q} \triangleq\left[\begin{array}{c}
\partial_{t} \mathbf{u}+\mathbf{u} \cdot \nabla \mathbf{u}+\nabla p-\nu \Delta \mathbf{u} \\
\nabla \cdot \mathbf{u}
\end{array}\right]=\left[\begin{array}{l}
\mathbf{f} \\
0
\end{array}\right], \\
\mathbf{u}(t=0, \mathbf{x})=\mathbf{u}_{\mathbf{0}}(\mathbf{x}),
\end{gathered}
$$

where the symbol $\triangleq$ stands for "equal by definition", $\mathbf{u}(t, \cdot): \mathbb{T}^{2} \rightarrow \mathbb{R}^{2}$ is the velocity field, $p(t, \cdot): \mathbb{T}^{2} \rightarrow \mathbb{R}$ is the pressure, $\nu$ is the coefficient of kinematic viscosity and $\mathbf{f}(t, \cdot): \mathbb{T}^{2} \rightarrow \mathbb{R}^{2}$ is the external forcing. The vector function $\mathbf{q}=\left[\begin{array}{ll}\mathbf{u} & p\end{array}\right]^{T}$ contains the two components of velocity field $\mathbf{u}$ and the pressure field $p$. For any solution of equation (2.1) we define the energy spectrum as

$$
E(t, k)=\frac{1}{2} \int_{\mathcal{C}(k)}|\hat{\mathbf{u}}(t, \mathbf{k})|^{2} \mathrm{~d} S(\mathbf{k}),
$$

where $\hat{\mathbf{u}}$ is the Fourier transform of $\mathbf{u}$ and $\mathbf{k}$ is the wave vector. $\mathcal{C}(k)$ is a circle with radius $k$ in 2-D plane, $\mathcal{C}(k)=\left\{\mathbf{k} \in \mathbb{R}^{2}:|\mathbf{k}|=k\right\}$.

Let $E_{0}(k)$ be the energy spectrum predicted by KLB theory, i.e.,

$$
E_{0}(k)= \begin{cases}C_{1} k^{-5 / 3} & k_{1}^{e} \leq k \leq k_{2}^{e} \\ C_{2} k^{-3} & k_{1}^{z} \leq k \leq k_{2}^{z}\end{cases}
$$




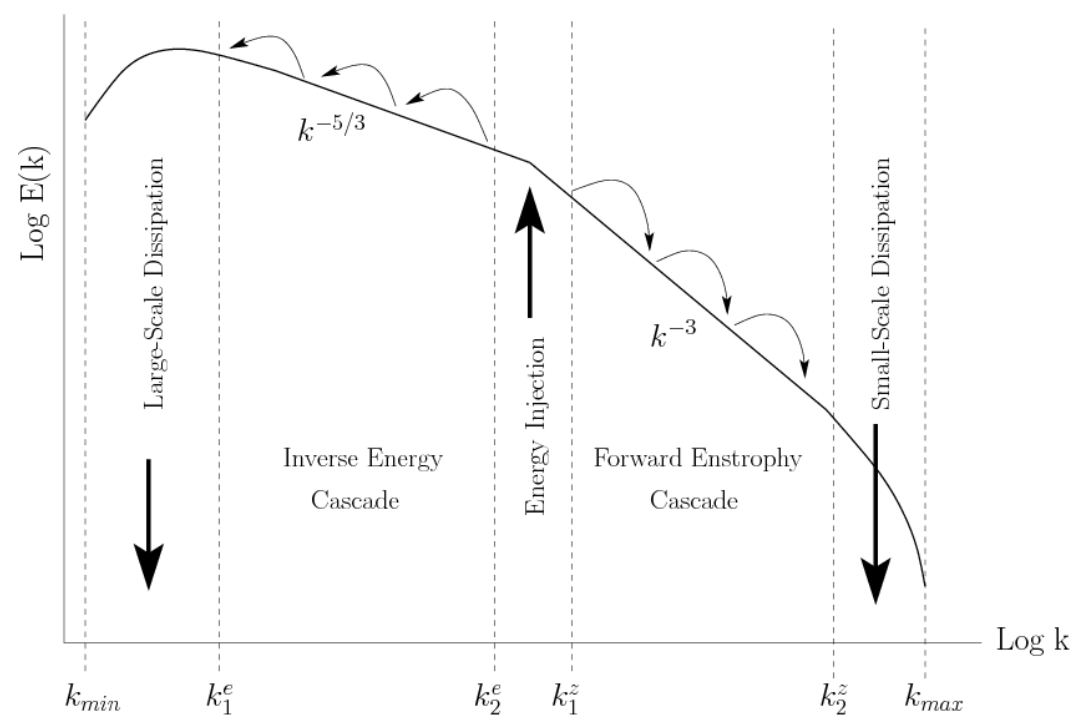

Figure 1: Schematic representation of the KLB theory. Energy and enstrophy are injected by the external forcing over the range $\left(k_{2}^{e}, k_{1}^{z}\right)$. Energy and estrophy inertial ranges are $\left[k_{1}^{e}, k_{2}^{e}\right]$ and $\left[k_{1}^{z}, k_{2}^{z}\right]$ respectively. The smallest wavenumber is $k_{\min }=1$ (if the domain is unbounded $k_{\min }=0$ and the large scale dissipation is not necessary) while the largest available wavenumber $k_{\max }$ depends on the numerical resolution.

where $\left[k_{1}^{e}, k_{2}^{e}\right]$ and $\left[k_{1}^{z}, k_{2}^{z}\right]$ are the energy and enstrophy inertial ranges respectively (figure 1). The wavenumber $k_{1}^{e}$ may extend to the smallest wavenumbers, $k_{2}^{z}$ extends up to the beginning of the dissipation range and $k_{2}^{e} \approx k_{1}^{z}$. In the case of monoscale forcing, $k_{2}^{e}$ and $k_{1}^{z}$ are almost equal to the forcing wavenumber. $C_{1}$ depends only on the energy dissipation rate $(\epsilon)$ and $C_{2}$ depends only on the enstrophy dissipation rate $(\eta)$. Since the energy and enstrophy dissipation rates are constants (based on KLB theory), $C_{1}$ and $C_{2}$ are also constants. Using the inertial range hypothesis, dimensional analysis gives $C_{1} \propto \epsilon^{2 / 3}$ and $C_{2} \propto \eta^{2 / 3}$. The constants of proportionality are non-dimensional and of order of unity (Constantin et al. 1994).

Our goal is to find a forcing, $\mathbf{f}$, which results in a solution of Navier-Stokes equation $(2.1)$ with the KLB energy spectrum $E_{0}(k)$. Define the following cost functional:

$$
\mathcal{J}(\mathbf{f}) \triangleq \frac{1}{2} \int_{0}^{T} \int_{I} w(t, k)\left|E(t, k)-E_{0}(k)\right|^{2} \mathrm{~d} k \mathrm{~d} t
$$

where $I=\left[k_{1}^{e}, k_{2}^{e}\right] \cup\left[k_{1}^{z}, k_{2}^{z}\right]$. The function $w(t, k)$ is a positive weight function which plays two roles. The wavenumber dependence of $w(t, k)$ normalizes the error $\left|E(t, k)-E_{0}(k)\right|^{2}$ over the range $I$ in order to get a uniform error distribution over all wavenumbers. Since $E_{0}(k)$ decreases as $k^{-3}$ on the interval $\left[k_{1}^{z}, k_{2}^{z}\right], w(t, k)=k^{6} \alpha(t)$ is a suitable candidate. The time dependence of the weight function $\alpha(t)$, on the other hand, is used to put more emphasis on the contribution of $E(t, k)$ near $t=T$. This allows the energy spectrum of the flow to evolve gradually from the initial energy spectrum $E(0, k)$ (which is arbitrary due to universality of the KLB theory) toward its equilibrium $E_{0}(k)$. Any increasing function of time would be an equally suitable choice for $\alpha(t)$.

If for some solution of equation (2.1), the above cost functional is zero, the energy spectrum will scale as predicted by the KLB theory on the time interval $[0, T]$. In this case, 
the energy spectrum will be stationary for times when $w(t, k)>0$. However, note that this does not imply, by itself, the existence of the dual cascades with the constant energy and enstrophy fluxes. The dynamics of the cascades must be examined independently.

The above description may be formulated as the following optimization problem

$$
\min _{\mathbf{f} \in \mathcal{U}} \mathcal{J}(\mathbf{f}),
$$

where $\mathcal{U}$ is a suitable function space with Hilbert structure. Here we consider the space of square integrable functions in space and time i.e., $\mathcal{U}=L^{2}\left([0, T] ; L^{2}\left(\mathbb{T}^{2}\right)\right)$. The cost functional $\mathcal{J}$ depends on $\mathbf{f}$ through the system of equations (2.1) in which the explicit dependence of the cost functional on the velocity field has been dropped. This type of cost functional is called a reduced cost functional (Nocedal \& Wright 2000).

Now, our goal is to find a forcing $\mathbf{f}_{\text {opt }} \in \mathcal{U}$ that minimizes the cost functional $\mathcal{J}$. Starting with an initial guess $\mathbf{f}^{(0)}$, an approximation of the minimizer can be found using a gradient-based descent method of the form

$$
\mathbf{f}^{(n+1)}=\mathbf{f}^{(n)}+\tau^{(n)} \mathcal{A} \nabla \mathcal{J}\left(\mathbf{f}^{(n)}\right), \quad n=0,1, \ldots,
$$

such that $\lim _{n \rightarrow \infty} \mathbf{f}^{(n)}=\mathbf{f}_{\text {opt }}$, where $n$ is the iteration count and $\tau^{(n)} \in \mathbb{R}^{-}$is a constant to be determined at each iteration. At each iteration, the descent direction $\mathcal{A} \boldsymbol{\nabla} \mathcal{J}$ is calculated based on the gradient of the cost functional $\nabla \mathcal{J}$. Different forms of the operator $\mathcal{A}$ correspond to different variants of the gradient method. For instance, if $\mathcal{A}$ is the identity operator, it corresponds to the steepest descent method and if it is an appropriate affine operator, it corresponds to the conjugate gradient method. As will be shown below, the gradient $\nabla \mathcal{J}$ may be expressed in terms of the solution of a suitably-defined adjoint system. This is a standard approach to solution of PDE-constrained optimization problems and its mathematical foundations were laid by Lions (1969). We refer the reader to the monographs by Gunzburger (2003) and Bewley (2001) for a survey of applications of this approach in fluid mechanics.

The necessary condition characterizing the minimizer $\mathbf{f}_{\text {opt }}$ of the cost functional is the vanishing of the Gâteaux differential $\mathcal{J}^{\prime}: \mathcal{U} \times \mathcal{U} \rightarrow \mathbb{R}$, i.e.,

$$
\mathcal{J}^{\prime}\left(\mathbf{f}_{\text {opt }} ; \mathbf{f}^{\prime}\right)=0, \quad \forall \mathbf{f}^{\prime} \in \mathcal{U},
$$

where the Gâteaux differential is defined as

$$
\mathcal{J}^{\prime}\left(\mathbf{f} ; \mathbf{f}^{\prime}\right) \triangleq \lim _{\epsilon \rightarrow 0} \frac{\mathcal{J}\left(\mathbf{f}+\epsilon \mathbf{f}^{\prime}\right)-\mathcal{J}(\mathbf{f})}{\epsilon} .
$$

Substituting from (2.2) and (2.4) into (2.8), one can easily show that

$$
\mathcal{J}^{\prime}\left(\mathbf{f} ; \mathbf{f}^{\prime}\right)=\frac{1}{2} \int_{0}^{T} \int_{I} w(t, k)\left(E(t, k)-E_{0}(k)\right)\left(\int_{\mathcal{C}(k)}\left(\widehat{\mathbf{u}} \cdot \overline{\widehat{\mathbf{u}}^{\prime}}+\overline{\widehat{\mathbf{u}}} \cdot \widehat{\mathbf{u}}^{\prime}\right) \mathrm{d} S(\mathbf{k})\right) \mathrm{d} k \mathrm{~d} t,
$$

where the bar represents the complex conjugate and $\widehat{\mathbf{u}}^{\prime}$ is the Fourier transform of the solution of the Navier-Stokes equation linearized around the state $\mathbf{u}$, i.e.

$$
\begin{gathered}
\mathcal{L}^{\prime} \mathbf{q}^{\prime} \triangleq\left[\begin{array}{c}
\partial_{t} \mathbf{u}^{\prime}+\mathbf{u}^{\prime} \cdot \boldsymbol{\nabla} \mathbf{u}+\mathbf{u} \cdot \nabla \mathbf{u}^{\prime}+\nabla p^{\prime}-\nu \Delta \mathbf{u}^{\prime} \\
\boldsymbol{\nabla} \cdot \mathbf{u}^{\prime}
\end{array}\right]=\left[\begin{array}{c}
\mathbf{f}^{\prime} \\
0
\end{array}\right], \\
\mathbf{u}^{\prime}(t=0, \mathbf{x})=0,
\end{gathered}
$$

where $\mathbf{q}^{\prime}=\left[\begin{array}{ll}\mathbf{u}^{\prime} & p^{\prime}\end{array}\right]^{T}$ and $\mathbf{f}^{\prime}$ is the direction in the space $\mathcal{U}$ in which the Gâteaux differential is computed in (2.8). 
On the other hand, the Riesz representation theorem (Lebedev \& Vorovich 2002) guarantees the existence of a unique element $\nabla \mathcal{J} \in \mathcal{U}$ which satisfies the identity

$$
\mathcal{J}^{\prime}\left(\mathbf{f} ; \mathbf{f}^{\prime}\right)=\left(\nabla \mathcal{J}, \mathbf{f}^{\prime}\right)_{\mathcal{U}}, \quad \forall \mathbf{f}^{\prime} \in \mathcal{U}
$$

where $(\cdot, \cdot) \mathcal{U}$ is the $L^{2}$ inner product. Hereafter, the subscript $\mathcal{U}$ is eliminated from the notation of the inner product. Here we consider the cost functional gradients $\nabla \mathcal{J}$ as $L^{2}$-functions, however, this approach can be easily generalized to different Hilbert spaces and, in particular, Sobolev spaces (Protas et al. 2004). Note that $\nabla \mathcal{J}$ is the steepest ascent direction for the cost functional $\mathcal{J}$. An expression for the gradient $\nabla \mathcal{J}$ cannot be derived imediately by equating (2.9) and (2.11), since in (2.9) the direction $\mathbf{f}^{\prime}$ does not appear explicitly as a factor (It is "hidden" in system (2.10) defining $\mathbf{u}^{\prime}$ ). However, the Gâteaux differential (2.9) can be transformed to the Riesz form using suitably-defined adjoint variables $\mathbf{u}^{*}$ and $p^{*}$, as shown by the following argument.

For any $\mathbf{q}^{*}=\left[\begin{array}{ll}\mathbf{u}^{*} & p^{*}\end{array}\right]^{T}$, we have $\left(\mathbf{u}^{*}, \mathbf{f}^{\prime}\right)=\left(\mathbf{q}^{*},\left[\begin{array}{c}\mathbf{f}^{\prime} \\ 0\end{array}\right]\right)$ by definition and $\left(\mathbf{q}^{*},\left[\begin{array}{c}\mathbf{f}^{\prime} \\ 0\end{array}\right]\right)=$ $\left(\mathbf{q}^{*}, \mathcal{L}^{\prime} \mathbf{q}^{\prime}\right)$ by $(2.10)$. Therefore,

$$
\begin{aligned}
\left(\mathbf{u}^{*}, \mathbf{f}^{\prime}\right) & =\left(\mathbf{q}^{*}, \mathcal{L}^{\prime} \mathbf{q}^{\prime}\right) \\
& =\left(\mathcal{L}^{*} \mathbf{q}^{*}, \mathbf{q}^{\prime}\right),
\end{aligned}
$$

where the adjoint operator $\mathcal{L}^{*}$ is

$$
\mathcal{L}^{*} \mathbf{q}^{*}=\left[\begin{array}{c}
-\partial_{t} \mathbf{u}^{*}-\left[\begin{array}{c}
\boldsymbol{\nabla} \mathbf{u}^{*}+\nabla \mathbf{u}^{*^{T}} \\
-\boldsymbol{\nabla} \cdot \mathbf{u}^{*}
\end{array}\right] \mathbf{u}-\nabla p^{*}-\nu \Delta \mathbf{u}^{*}
\end{array}\right]
$$

which was obtained by integration by parts. Note that the boundary terms resulting from integration by parts in space cancel out due to the periodic boundary conditions. Assuming $\mathbf{u}^{*}(t=T, \mathbf{x})=\mathbf{0}$, the terms $\left.\int_{\mathbb{T}^{2}} \mathbf{u}^{*} \cdot \mathbf{u}^{\prime}\right|_{t=T} \mathrm{~d} \mathbf{x}$ and $\left.\int_{\mathbb{T}^{2}} \mathbf{u}^{*} \cdot \mathbf{u}^{\prime}\right|_{t=0} \mathrm{~d} \mathbf{x}$, resulting from integration by parts in time, also vanish since $\mathbf{u}^{\prime}(t=0, \mathbf{x})=\mathbf{0}$.

By Parseval's identity,

$$
\begin{aligned}
\left(\mathcal{L}^{*} \mathbf{q}^{*}, \mathbf{q}^{\prime}\right) & =\left(\widehat{\mathcal{L}^{*} \mathbf{q}^{*}}, \widehat{\mathbf{q}}^{\prime}\right) \\
& =\int_{0}^{T} \int_{0}^{\infty}\left(\int_{\mathcal{C}(k)} \widehat{\mathcal{L}^{*} \mathbf{q}^{*}} \cdot \overline{\widehat{\mathbf{q}}^{\prime}} \mathrm{d} S(\mathbf{k})\right) \mathrm{d} k \mathrm{~d} t,
\end{aligned}
$$

where the hat represents the Fourier transform. Since $\left(\mathcal{L}^{*} \mathbf{q}^{*}, \mathbf{q}^{\prime}\right)$ is real valued, $\left(\mathcal{L}^{*} \mathbf{q}^{*}, \mathbf{q}^{\prime}\right)=$ $\frac{1}{2}\left(\left(\widehat{\mathcal{L}^{*} \mathbf{q}^{*}}, \widehat{\mathbf{q}}^{\prime}\right)+\widehat{\left(\widehat{\mathcal{L}^{*} \mathbf{q}^{*}}, \widehat{\mathbf{q}}^{\prime}\right)}\right)$. Therefore,

$$
\left(\mathcal{L}^{*} \mathbf{q}^{*}, \mathbf{q}^{\prime}\right)=\frac{1}{2} \int_{0}^{T} \int_{0}^{\infty}\left(\int_{\mathcal{C}(k)}\left(\widehat{\mathcal{L}^{*} \mathbf{q}^{*}} \cdot \overline{\widehat{\mathbf{q}}^{\prime}}+\overline{\mathcal{L}^{*} \mathbf{q}^{*}} \cdot \widehat{\mathbf{q}}^{\prime}\right) \mathrm{d} S(\mathbf{k})\right) \mathrm{d} k \mathrm{~d} t .
$$

A comparison between (2.9) and (2.15) suggests that if the following choice is made for the right-hand side expression in the equation for the adjoint variable $\mathbf{q}^{*}$ :

$$
\widehat{\mathcal{L}^{*} \mathbf{q}^{*}}(t, \mathbf{k})=\left[\begin{array}{c}
\chi_{I}(k) w(t, k)\left(E(t, k)-E_{0}(k)\right) \hat{\mathbf{u}}(t, \mathbf{k}) \\
0
\end{array}\right]
$$

with $\chi_{I}$ the characteristic function of the interval $I$, then the adjoint operator can be used to re-express Gâteaux differential $(2.9)$ as $\mathcal{J}^{\prime}\left(\mathbf{f} ; \mathbf{f}^{\prime}\right)=\left(\mathcal{L}^{*} \mathbf{q}^{*}, \mathbf{q}^{\prime}\right)$. This, together with the Riesz identity (2.11) and the duality expression (2.12) implies that $\mathcal{J}^{\prime}\left(\mathbf{f} ; \mathbf{f}^{\prime}\right)=\left(\mathbf{u}^{*}, \mathbf{f}^{\prime}\right)=$ $\left(\nabla \mathcal{J}, \mathbf{f}^{\prime}\right)$ for any $\mathbf{f}^{\prime} \in \mathcal{U}$ and therefore,

$$
\nabla \mathcal{J}=\mathbf{u}^{*}
$$


Hence, the gradient direction $\nabla \mathcal{J}$ can be conveniently expressed in terms of the solution of the following adjoint system

$$
\begin{gathered}
\left(\partial_{t} \mathbf{u}^{*}+\left[\boldsymbol{\nabla} \mathbf{u}^{*}+\nabla \mathbf{u}^{*^{T}}\right] \mathbf{u}+\nabla p^{*}+\nu \Delta \mathbf{u}^{*}\right)^{\wedge}(t, \mathbf{k})=-\chi_{I}(k) w(t, k)\left(E(t, k)-E_{0}(k)\right) \widehat{\mathbf{u}}(t, \mathbf{k}), \\
\boldsymbol{\nabla} \cdot \mathbf{u}^{*}=0 \\
\mathbf{u}^{*}(t=T, \mathbf{x})=0 .
\end{gathered}
$$

By solving the adjoint system to compute the gradient $\nabla \mathcal{J}$ and using the iterative process (2.6), one can find an approximation of the minimizer $\mathbf{f}_{\text {opt }}$. The optimal value for the parameter $\tau^{(n)}$ in (2.6) is the one that minimizes the function $\mathcal{G}(\tau) \triangleq$ $\mathcal{J}\left(\mathbf{f}^{(n)}+\tau \mathcal{A} \boldsymbol{\nabla} \mathcal{J}\left(\mathbf{f}^{(n)}\right)\right)$ with respect to the real variable $\tau$ for fixed $\mathbf{f}^{(n)}$ and $\mathcal{A} \boldsymbol{\nabla} \mathcal{J}\left(\mathbf{f}^{(n)}\right)$. Here a standard line minimization method (Nocedal \& Wright 2000) is used to find the appropriate value of $\tau$ at each iteration.

Optimization problems of type (2.5) are examples of inverse problems which often tend to be ill-posed. This ill-posedness may manifest itself in large magnitudes of the minimizer $\mathbf{f}_{\mathrm{opt}}$. Since such large-magnitude forcing is not very interesting, this problem can be mitigated by adding a penalty term to the cost functional as follows

$$
\mathcal{J}_{\eta}(\mathbf{f})=\mathcal{J}(\mathbf{f})+\frac{1}{2} \eta\|\mathbf{f}\|^{2}
$$

where $\mathcal{J}(\mathbf{f})$ is the same as in (2.4), $\eta \in \mathbb{R}^{+}$is a constant and $\|\cdot\|$ is the norm in $\mathcal{U}$ $\left(L^{2}\right.$-norm here). The penalty on the magnitude of the control variable $\mathbf{f}$ is determined by the parameter $\eta$. Smaller values of $\eta$ allow forcings with larger norms and vice versa. Since the Gâteaux differential of the penalty term $\frac{1}{2} \eta\|\mathbf{f}\|^{2}$ in direction $\mathbf{f}^{\prime}$ is $\eta\left(\mathbf{f}, \mathbf{f}^{\prime}\right) \mathcal{U}$, the gradient of the modified cost functional is $\boldsymbol{\nabla} \mathcal{J}_{\eta}(\mathbf{f})=\boldsymbol{\nabla} \mathcal{J}+\eta \mathbf{f}$.

To summarize, the optimization process can be expressed as the following algorithm.

Algorithm 1.

(1) Choose an initial guess $\mathbf{f}^{(0)}(t, \mathbf{x})$; Set $n=0$.

(2) Solve Navier-Stokes equation (2.1) forward in time with $\mathbf{f}=\mathbf{f}^{(n)}$.

(3) Solve adjoint equation (2.18) backward in time.

(4) Obtain the cost functional gradient as $\nabla \mathcal{J}_{\eta}=\mathbf{u}^{*}+\eta \mathbf{f}$

(5) Find the length of the step $\tau^{(n)}$ through line minimization.

(6) Update the control variable through (2.6); Set $n=n+1$.

(7) Go back to 2 .

The loop continues until the optimality condition (2.7) is approximately satisfied, i.e., $\nabla \mathcal{J}_{\eta}\left(\mathbf{f}^{(n)}\right) \approx 0$ in some suitable sense.

In this work, a pseudo-spectral method is used to solve Navier-Stokes and adjoint equations numerically. Since the adjoint equation is expressed in terms of the Fourier space representatives and the boundary conditions are doubly periodic, the Fourier spectral method is arguably the most efficient way to solve it. Time integration is performed with a Krylov subspace method (see Edwards et al. 1994; Schulze et al. 2009). As was mentioned above, the parameter $\tau^{(n)}$ is evaluated by a line search method. This method uses successive evaluations of the cost functional. Each evaluation of the cost functional requires solving the Navier-Stokes equation. Usually between 15 to 25 cost functional evaluations are required for each line minimization. Therefore, it turns out that the most costly part of the above algorithm is evaluation of the parameter $\tau^{(n)}$.

In the next section, we use this control method to study the effects of forcing on the scaling properties of the energy spectrum in forced $2-\mathrm{D}$ turbulence. Since to the best of 
our knowledge adjoint-based techniques have not been employed to control the spectral properties of a turbulent flow, this study serves also as a validation for the control method proposed here.

\section{Results and Discussion}

Equations (2.1) and (2.18) are solved with $\nu=6.5 \times 10^{-5}$ using $1024^{2}$ Fourier modes. The aliasing effect of the nonlinear term is removed by the $2 / 3$ rule. To reduce the computational time, the initial condition, $\mathbf{u}_{\mathbf{0}}$, is a fully-developed turbulent field forced by a mono-scale forcing (see figure 2). The initial guess in step 1 of the algorithm 1 is zero, i.e. $\mathbf{f}^{(0)} \equiv \mathbf{0}$. The constants of proportionality in equation (2.3) are $C_{1}=1.18$ and $C_{2}=64.00$. These values are chosen in order to retrieve a continuous target energy spectrum, $E_{0}(k)$, with the total energy close to the total energy of the initial condition. The weight function in equation $(2.4)$ is $w(t, k)=k^{6} \sqrt{t / T}$ in order to normalize the error over different wave-numbers and also to put more emphasis on the contribution of the error near $t=T$, where the termination time is $T=4$. Here, the penalization parameter $\eta$ in (2.19) is equal to zero.

Based on the types of forcing, the results are divided into two parts as follows:

(i) Full-band forcing $(\S 3.1)$

(ii) Band-limited forcing $(\S 3.2)$

In case (i) the forcing is allowed to be active at any wavenumber. Therefore, it does not necessarily respect the inertial range assumption of the KLB theory since it may add (or remove) energy and enstrophy into (from) the cascading wavenumbers. We show, however, that the inertial interactions (responsible for the cascades) still dominate the flow dynamics. This forcing is physically more realistic than the (theoretically interesting) monoscale forcing. For instance, Welch \& Tung (1998) present a model of the atmospheric flow in which the forcing is due to temperature gradients and active over a portion of the enstrophy cascade.

In case (ii) the forcing is non-zero only at a few intermediate wavenumbers (i.e. $k \in\left(k_{2}^{e}, k_{1}^{z}\right)$ ) and a few small wavenumbers (i.e. $k \in\left[1, k_{1}^{e}\right)$ ) (see figure 1 ). Therefore, the scalings of the energy and enstrophy cascades are exclusively due to inertial (triad) interactions. This forcing resembles the classical band-limited forcing used in most numerical simulations. However, we do not define the forcing a priori, but calculate it through the optimization. Note that neither a linear damping nor a hypoviscosity is imposed as a large scale sink. Instead, we simply allow the forcing to be non-zero at a few small wavenumbers (i.e. $k \in\left[1, k_{1}^{e}\right)$ ) in order to allow for large scale energy dissipation, if necessary. As shown in the next section, this large-scale sink of energy is indeed created by the forcing.

In terms of the optimal control setting, in case (i) the only restriction on the forcing is to be square integrable. In other words the cost functional $(\mathcal{J})$ is minimized over the function space $\mathcal{U}=L^{2}\left([0, T] ; L^{2}\left(\mathbb{T}^{2}\right)\right)$. While in case (ii), the control space is

$$
\mathcal{U}=\left\{\mathbf{f} \in L^{2}\left([0, T] ; L^{2}\left(\mathbb{T}^{2}\right)\right): \hat{\mathbf{f}}(t, \mathbf{k})=\mathbf{0},|\mathbf{k}| \in\left[k_{1}^{e}, k_{2}^{e}\right] \cup\left[k_{1}^{z},+\infty\right)\right\}
$$

\subsection{Full-band Forcing}

The parameters that determine the scaling ranges are $k_{1}^{e}=2, k_{2}^{e}=k_{1}^{z}=20$ and $k_{2}^{z}=200$. Figure 2 shows that the optimal control method gives the spectral slopes predicted by KLB theory. This energy spectrum remains (almost) stationary on the time interval $\frac{1}{2} T \leq t<T$ where $T=4 \approx$ two eddy turnover times. In figure 3 , the instantaneous vorticity fields produced by a monoscale forcing (left) and the optimal forcing (right) are 


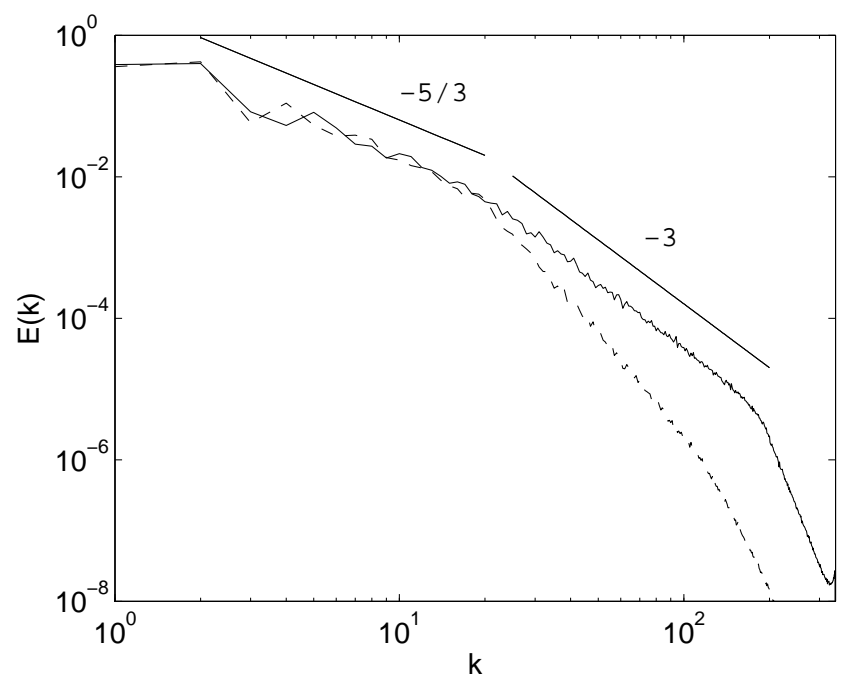

Figure 2: The energy spectrum of the initial condition (- - ) and the energy spectrum resulting from the optimal full-band forcing $(-)$. The straight lines represent the $-5 / 3$ and -3 slopes.

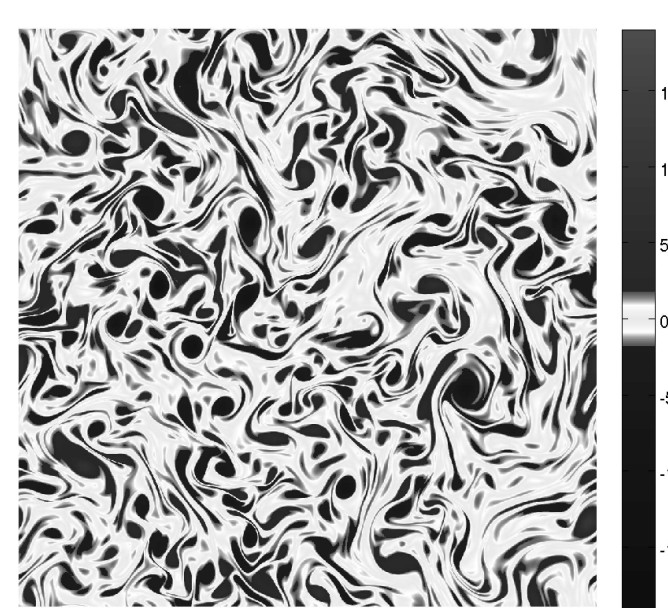

(a)

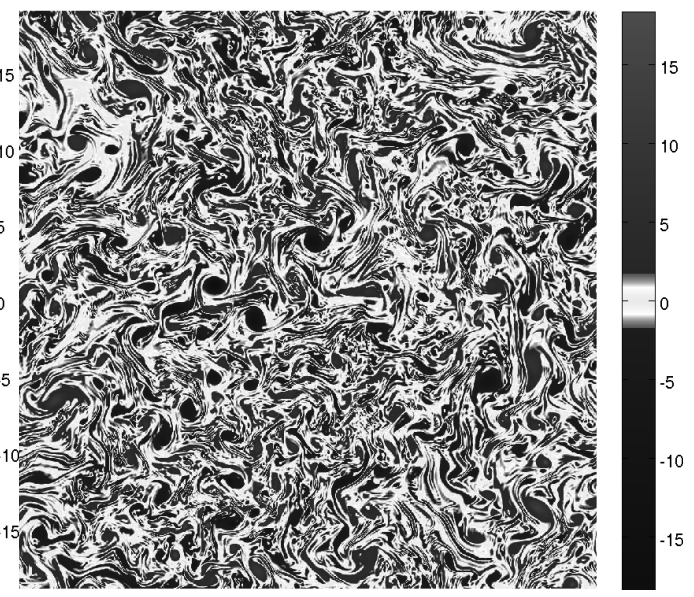

(b)

Figure 3: Vorticity fields resulting from the band-limited forcing (a) and the optimal full-band forcing (b)

compared. It is obvious that the optimal forcing produces more small scale, filamentary structures.

We now present some properties of this optimal forcing. An interesting quantity is the contribution of the forcing to the energy spectrum of the velocity field. This quantity is defined by

$$
F(t, k)=\int_{|\mathbf{k}|=k} \Re\{\hat{\mathbf{f}}(t, \mathbf{k}) \cdot \overline{\hat{\mathbf{u}}(t, \mathbf{k})}\} \mathrm{d} S(\mathbf{k}),
$$




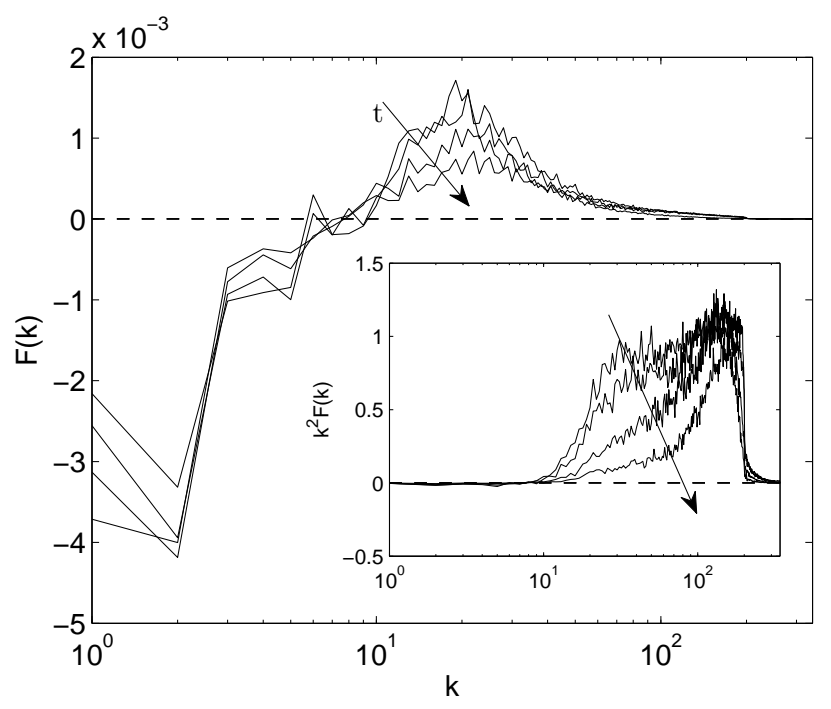

Figure 4: The energy is mostly injected at $k=20$ by the external forcing. Different lines correspond to different time slices. The inset shows the corresponding enstrophy injection.

and indicates whether the forcing injects energy into (if positive) or removes energy from (if negative) mode $k$. Figure 4 shows that the optimal control injects energy into the system mostly at wave-number $k=20$ (i.e. the wave-number at which the spectral slope changes from $-5 / 3$ to -3 , see figure 2 ). This is a non-trivial result since the control method allows the forcing to act over the whole wavenumber range. The energy injection by forcing decays to zero exponentially for $k \geq 20$. Enstrophy, however, is mostly injected at small scales as the inset of figure 4 shows. For $1 \leq k \leq 6$ the optimal forcing removes energy from the system which creates a sink of energy in large scales. Since energy is transferred to larger scales, a mechanism to dissipate it is necessary in order to reach a statistically stationary state in forced 2-D turbulence. Moreover, Tran \& Shepherd (2002) proved that the presence of a large scale sink of energy is necessary in order to obtain the dual cascades with $-5 / 3$ and -3 slopes when the forcing is monoscale or band-limited. In the present case where the forcing is full-band the large scale energy dissipation seems to be necessary, and is produced automatically by the optimal control method.

The energy spectrum of the forcing which is defined by

$$
E_{f}(t, k)=\frac{1}{2} \int_{|\mathbf{k}|=k}|\hat{\mathbf{f}}(t, \mathbf{k})|^{2} \mathrm{~d} S(\mathbf{k}),
$$

is plotted in figure 5 for several time slices. It shows that the forcing is active on a wide range of scales which means that the energy and enstrophy cascades are not inertial ranges. The forcing decays to zero as time increases (also consistent with figure 4). This decay is an artifact of the control algorithm. Since we start with a zero initial guess (i.e. $\mathbf{f}^{(0)} \equiv \mathbf{0}$ ) and the gradient of the cost functional is zero at $t=T$ (see equation (2.18)), the forcing remains equal to zero at $t=T$ for all iterations, i.e. $\mathbf{f}^{(n)}(T, \mathbf{x}) \equiv \mathbf{0}$.

It is also necessary to examine the dynamical properties of the flow generated by the optimal forcing since one can generate a random phase vector field with -3 (or $-5 / 3$ ) energy spectrum (and no dynamics or cascades). Therefore, we need to check that the the 


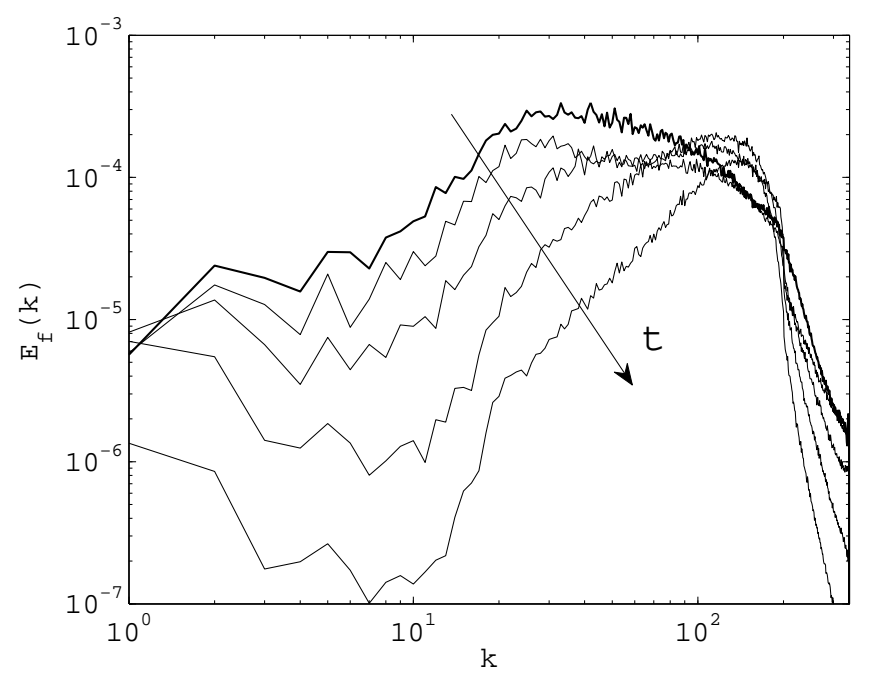

Figure 5: The energy spectrum of the forcing at different time slices. As time increases the energy level of the forcing decreases to zero.

resulting flow is dynamically active. As mentioned earlier, the triad interactions determine the dynamics of the energy and enstrophy cascades associated with the nonlinear term in the Navier-Stokes equation. In 2-D, they transfer most of the energy to larger scales and most of the enstrophy to smaller scales. Since our optimal forcing is non-zero on the cascading ranges, it can significantly affect these transfers. In the following, we will show that the triad interactions still dominate the dynamics of the flow in the presence of the optimal control forcing.

For each wave vector triad $\mathbf{k}, \mathbf{p}$ and $\mathbf{q}$, we use the method introduced in Maltrud \& Vallis (1993) to calculate the energy transfer function $T_{\mathbf{k p q}}$. The enstrophy transfer function, $S_{\mathbf{k p q}}$, is related to energy transfer function by $S_{\mathbf{k p q}}=k^{2} T_{\mathbf{k p q}}$. The positive values of the quantity $T_{\mathbf{k p q}}\left(S_{\mathbf{k p q}}\right)$ correspond to energy (enstrophy) transfer rate into mode $\mathbf{k}$ due to interactions with modes $\mathbf{p}$ and $\mathbf{q}$. Similarly, the negative values of these quantities correspond to the energy and enstrophy transfer rates out of mode $\mathbf{k}$.

We simplify the transfer functions (and make them consistent with the statistical KLB theory) by averaging over one of the wave-vectors and defining

$$
T_{\mathbf{k p}}=\sum_{\mathbf{q}} T_{\mathbf{k p q}}
$$

Since we consider isotropic turbulence, it is appropriate to average over angles in wavenumber space. This further simplifies the calculations and leads to the following definition of the energy transfer function in terms of two wave-numbers

$$
T_{k p}=\int_{|\mathbf{k}|=k} \int_{|\mathbf{p}|=p} T_{\mathbf{k p}} \mathrm{d} S(\mathbf{p}) \mathrm{d} S(\mathbf{k}) .
$$

The two-wave-number enstrophy transfer function, $S_{k p}$, is defined similarly, and is related to $T_{k p}$ by $S_{k p}=k^{2} T_{k p}$.

The time-averaged energy and enstrophy transfer rates are plotted in figure 6 for the fixed wave-number $k=10$ (for energy) and $k=40$ (for enstrophy) and in terms of $p$. The 


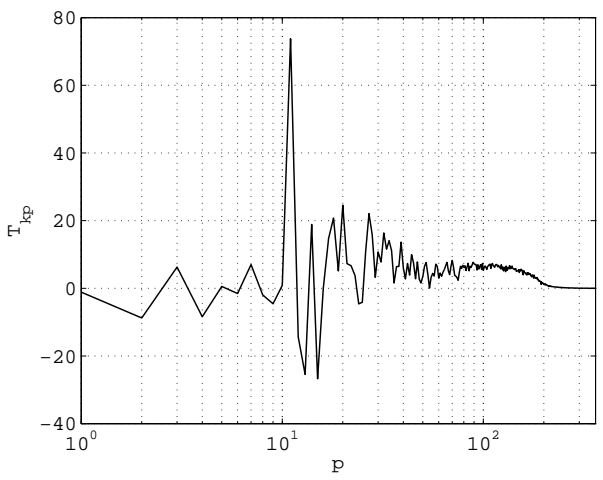

(a) $T_{k p}, k=10$.

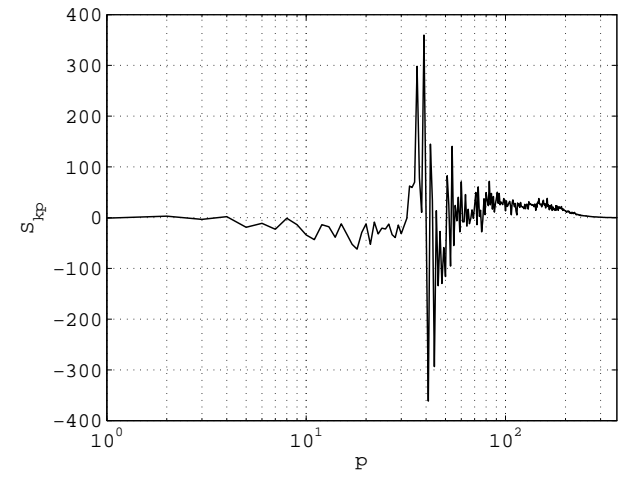

(b) $S_{k p}, k=40$

Figure 6: The time averaged energy $\left(T_{k p}\right)$ and enstrophy $\left(S_{k p}\right)$ transfer functions.

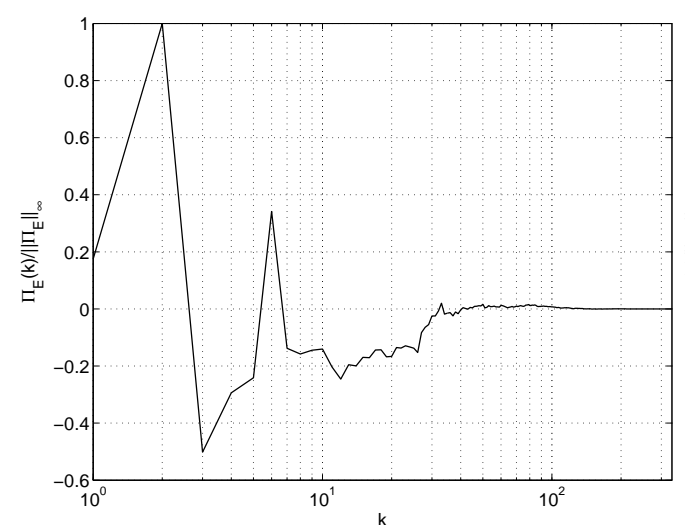

(a) Energy flux

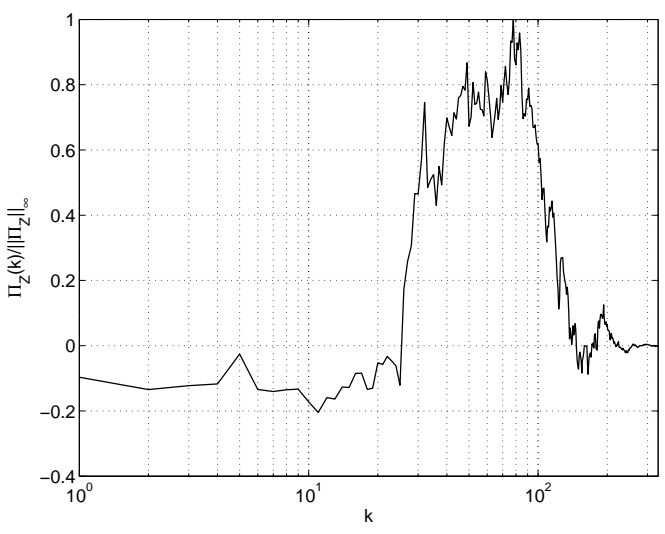

(b) Enstrophy flux

Figure 7: The time averaged fluxes of energy(a) and enstrophy(b) normalized by $\left\|\Pi_{E}\right\|_{\infty}=\sup _{k}\left|\Pi_{E}(k)\right|$ and $\left\|\Pi_{Z}\right\|_{\infty}=\sup _{k}\left|\Pi_{Z}(k)\right|$ respectively.

data is noisy since the transfer functions are averaged over a relatively short time interval $(0 \leq t \leq 4)$. However, some interesting features may be observed. In a neighbourhood of $k=40$, the enstrophy transfer function is positive for $p<k$ while it is negative for $p>k$. Similar behaviour is observed for wave-numbers $25 \leq k \leq 150$ (not presented here). This shows that the enstrophy is mostly transferred to smaller scales on this range of wave-numbers. On the other hand, in a small neighborhood of the wave-number $k$, the energy transfer function is negative for $p<k$ and positive for $p>k$ which shows an energy transfer to larger scales. These results provide evidence that the optimal forcing respects the directions of the energy and enstrophy cascades.

The energy and enstrophy fluxes

$$
\Pi_{E}(k)=-\int_{k}^{\infty} \int_{0}^{\infty} T_{\kappa p} \mathrm{~d} p \mathrm{~d} \kappa, \quad \Pi_{Z}(k)=-\int_{k}^{\infty} \kappa^{2} \int_{0}^{\infty} T_{\kappa p} \mathrm{~d} p \mathrm{~d} \kappa,
$$

show the flux of energy and enstrophy across wavenumber $k$ (see Maltrud \& Vallis 1993, 


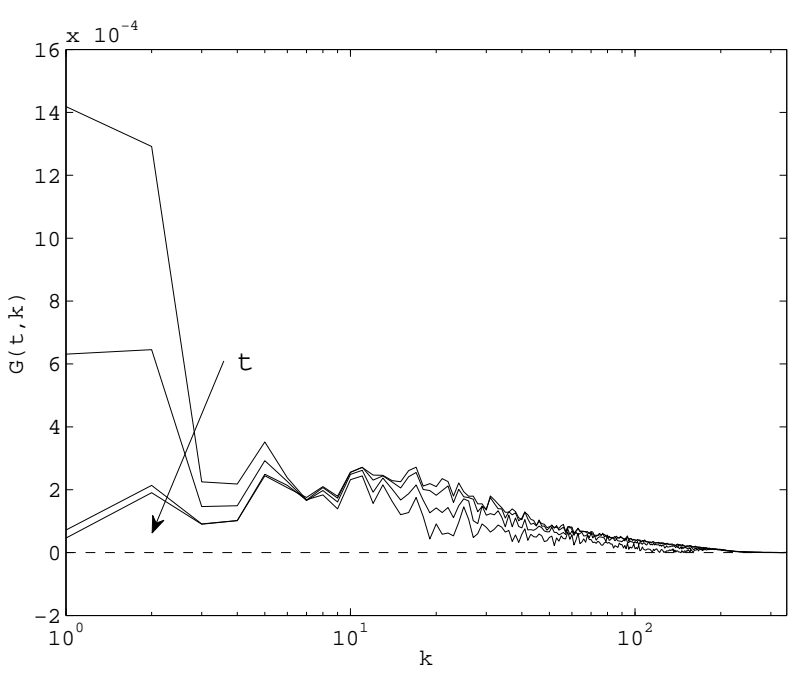

Figure 8: The fact that $G(t, k)$ is positive for all $k$ demonstrates the existence of inverse energy and direct enstrophy cascades. Each curve corresponds to a time slice between $\frac{3}{4} T$ and $T$. The arrow shows the time increase.

for details). Figure 7 shows the time averaged fluxes normalized by the maximum of their absolute values. Since $\Pi_{E}(k)<0$ for $k \in[3,30)$ (except $k=6$ ) the energy fluxes are upscale on average. The dominant downscale flux of enstrophy is clear for $k \in(15,150)$ since $\Pi_{Z}(k)>0$ over this range.

There is another independent way of checking the direction of energy and enstrophy cascades. In their proof, Gkioulekas \& Tung (2007) present a sufficient condition for the existence of the upscale energy and downscale enstrophy cascades in a statistically stationary state. This condition is

$$
G(t, k) \triangleq 2 \nu k^{2} E(t, k)-F(t, k)>0
$$

where $E(t, k)$ is the energy spectrum as defined in $(2.2)$ and $F(t, k)$ is the energy injected (or removed) by the forcing calculated through (3.2). Note that the inequality holds for band-limited forcings and any wave-number, $k$, outside the band-width of the forcing since $F(t, k)=0$ for these modes. Figure 8 shows the quantity $G(t, k)$ for the optimal forcing and for several time slices. Note that the time dependence of the energy spectrum can be eliminated since a statistically steady state is considered, however, $F(t, k)$ is still time dependent. This figure shows that the inequality (3.4) is satisfied and therefore, on average, energy is transferred to larger scales while enstrophy is transferred to smaller scales.

In summary, our results demonstrate the existence of a forcing which is consistent with the predictions of KLB theory (i.e. coexisting cascades of energy and enstrophy with $-5 / 3$ and -3 spectral slopes). The upscale energy and downscale enstrophy cascades are active under this forcing. However, these cascades are not inertial ranges since the forcing is active on a wide range of scales including the cascade ranges. The forcing injects energy mostly around the wave-number at which the slope of the energy spectrum changes from $-5 / 3$ to -3 . Moreover, it automatically removes energy from large scales and produces a statistically steady state. 


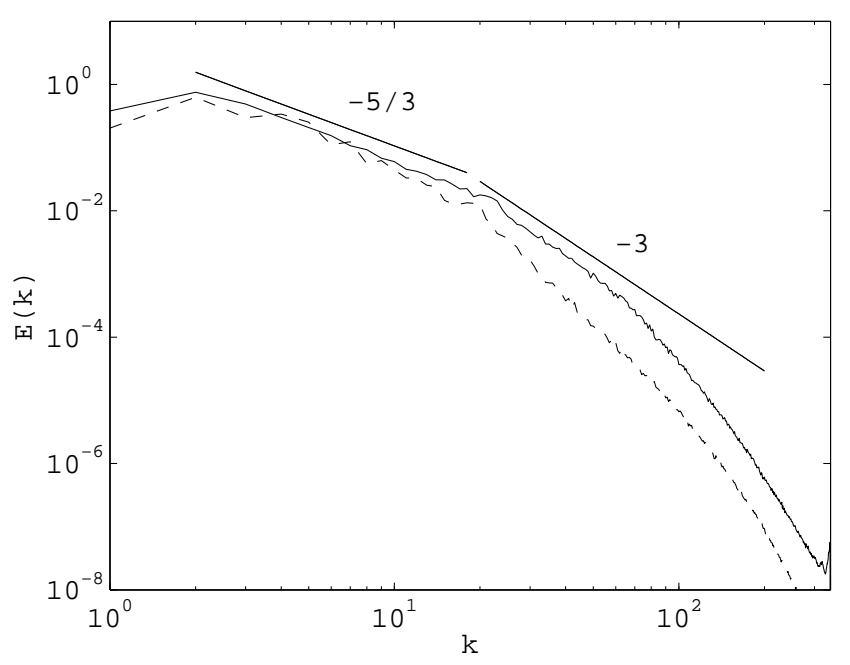

Figure 9: The controlled energy spectrum (with band-limited forcing) at $t=\frac{7}{8} T$ () and the energy spectrum resulting from the conventional band-limited forcing and inverse viscosity (- - ).

\subsection{Band-limited Forcing}

We now confine the forcing to the space of band-limited functions defined in (3.1). Since $k_{1}^{e}=3, k_{2}^{e}=18, k_{1}^{z}=25$ and $k_{2}^{z}=200$, the forcing is non-zero only for the wavenumbers $k \in[1,2] \cup[19,24]$. Note that conventionally, energy is removed from large scales by Ekman drag or by inverse viscosity. Here, we do not use any energy dissipative mechanisms at large scales. Instead, we simply allow the forcing to be non-zero at largest scales. The forcing may remove the energy from those scales if necessary (As the following results demonstrate, this is in fact the case). The advantage of this method is that the energy injection and large scale energy dissipation are now determined by the control procedure alone: they are not fixed a priori.

Figure 9 shows the energy spectrum resulting from the band-limited optimal forcing at $t=3.5$ (Note that in this case $T=4 \approx$ four eddy turnover times). The energy spectrum follows the KLB scaling law $E \sim k^{-5 / 3}$ for a decade of wave-numbers and $E \sim k^{-3}$ for a quarter of a decade. As expected, the enstrophy range is extended over a shorter range of wave-numbers compared to the previous case where full-band forcing was used. The energy spectrum resulting from a conventional band-limited forcing and inverse viscosity is given for comparison (dashed line in figure 9).

The vorticity field at $t=T$ is presented in figure 10a. Figure 10b shows the instantaneous non-zero component of $\boldsymbol{\nabla} \times \mathbf{f}$ in physical space. It appears that the forcing is homogeneous and isotropic. However, more careful investigation shows that the forcing is particularly aligned in the favour of enstrophy injection into the system. To see this, note that the total energy and enstrophy injections are given by $\int_{\mathbb{T}^{2}} \mathbf{f} \cdot \mathbf{u} \mathrm{d} \mathbf{x}$ and $\int_{\mathbb{T}^{2}} \mathbf{f} \cdot(-\Delta \mathbf{u}) \mathrm{d} \mathbf{x}$ respectively. Therefore, a forcing aligned with velocity injects energy more efficiently while a forcing aligned with $-\Delta \mathbf{u}$ injects enstrophy more efficiently. Figure 11 shows the probability distribution function of these alignments over time and space where $\theta_{e}$ and $\theta_{z}$ are respectively the distributions of the angles $\angle(\mathbf{f}, \mathbf{u})$ and $\angle(\mathbf{f},-\Delta \mathbf{u})$. These figures reveal that the forcing is aligned such that the enstrophy injection is rela- 


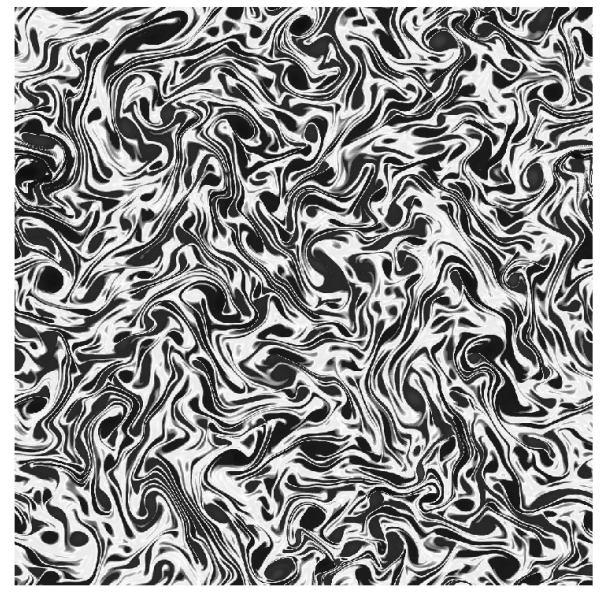

(a) $(\nabla \times \mathbf{u}) \cdot \widehat{\mathbf{n}}$

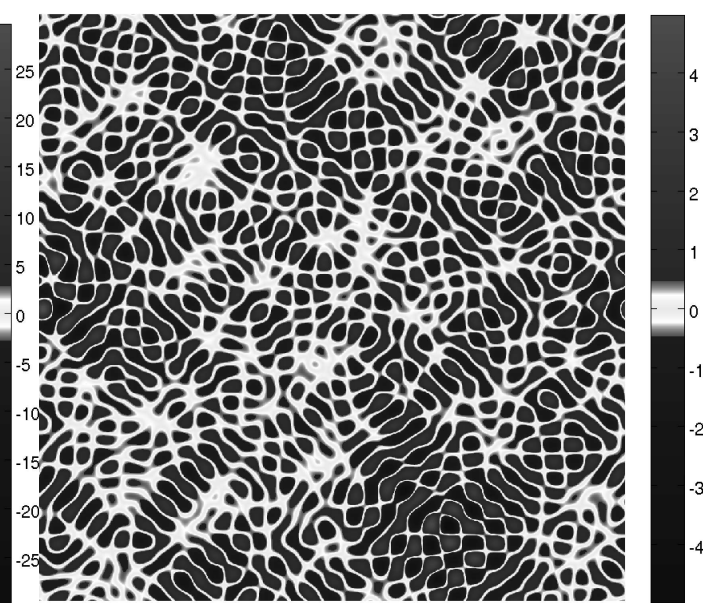

(b) $(\boldsymbol{\nabla} \times \mathbf{f}) \cdot \widehat{\mathbf{n}}$.

Figure 10: The vorticity field resulting from the optimal band-limited forcing (left) and the curl of the optimal forcing (right) at $t=\frac{7}{8} T$.

tively more efficient than the energy injection. Note that in the case of a random phase forcing (which is conventional in numerical simulations of 2-D turbulence) there are no preferential alignments of the forcing with the velocity field. Figure 12 shows the time correlation of the optimal forcing defined as $\langle\mathbf{f}(\mathbf{x}, t) \cdot \mathbf{f}(\mathbf{x}, t+\tau)\rangle$ where \langle\rangle denotes the average in time and space. The time correlations of the velocity and the deformation tensor $D_{i j}=\frac{1}{2}\left(\frac{\partial u_{i}}{\partial x_{j}}+\frac{\partial u_{j}}{\partial x_{i}}\right)$ are given for comparison. The time correlation of all elements of the deformation tensor (almost) coincide and therefore only one of them (non-diagonal element) is plotted. The curves are normalized by the correlation at $\tau=0$. It shows that the time correlation of the optimal forcing is relatively small and almost equal to the time correlation of the strain rate. This suggest a connection between the time dependence of the forcing and the strain rate.

The energy contribution to the system from the external forcing (i.e. $F(t, k)$ ) is shown in figure 13. Energy is injected in the wave-numbers $k \in[19,24]$, while it is removed from largest available scales, i.e. $k \in[1,2]$. This agrees with the fact that a sink of energy at large scales is a necessary condition for achieving the KLB limit in a finite domain. Since the forcing is band-limited, the inequality (3.4) is automatically satisfied on the range $k \in[3,18] \cup[25,+\infty)$. Therefore, the upscale energy and downscale enstrophy fluxes are dominant.

\section{Conclusions}

We have developed an optimal control method in order to study the effect of forcing on the scaling laws of two-dimensional turbulence. We are particularly interested in discovering whether it is possible to produce the simultaneous dual cascades of energy and enstrophy and the corresponding scaling laws (i.e. $E \sim k^{-5 / 3}$ on the energy cascade and $E \sim k^{-3}$ on the enstrophy cascade) predicted by the classical KLB theory in a non-asymptotic sense.

Our results demonstrate that when a full-band forcing is used (i.e. a forcing active over all scales), the KLB spectral slopes can be observed in a flow with a moderate Reynolds 


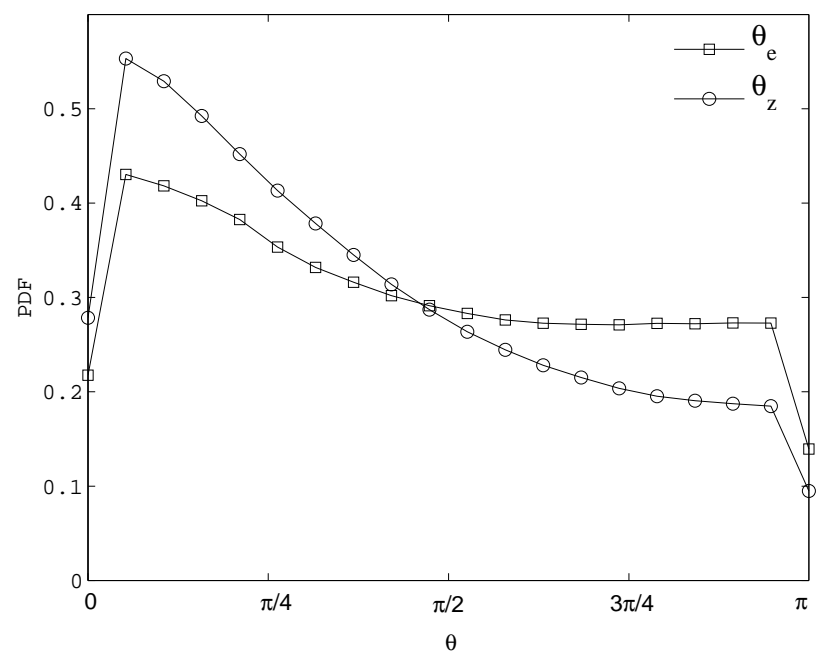

Figure 11: The probability distribution function of $\theta_{e}$ (the angle between $\mathbf{f}$ and $\mathbf{u}$ ) and $\theta_{z}$ (the angle between $\mathbf{f}$ and $-\Delta \mathbf{u}$ ).

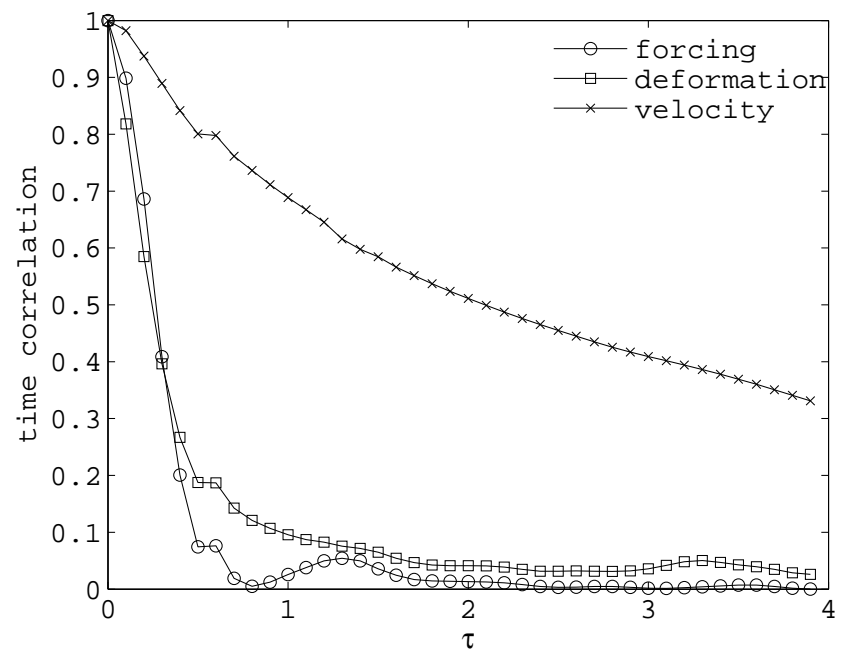

Figure 12: Normalized time-correlation functions.

number. Such forcings clearly violate the inertial range assumption because some energy is directly injected by the external forcing into the wave-numbers of the scaling ranges. However, the inverse energy and forward enstrophy cascades still exist and their dynamics are dominated by the inertial interactions (i.e. triad interactions). This is similar to the linear forcing suggested by Lundgren (2003) for numerical simulations of 3-D turbulence. Linear forcing is also full-band and therefore interferes with the inertial range dynamics. However, as we have found here for 2-D turbulence, Rosales \& Meneveau (2005) showed that the resulting statistical properties (e.g. stationarity and power-law scaling) of the 


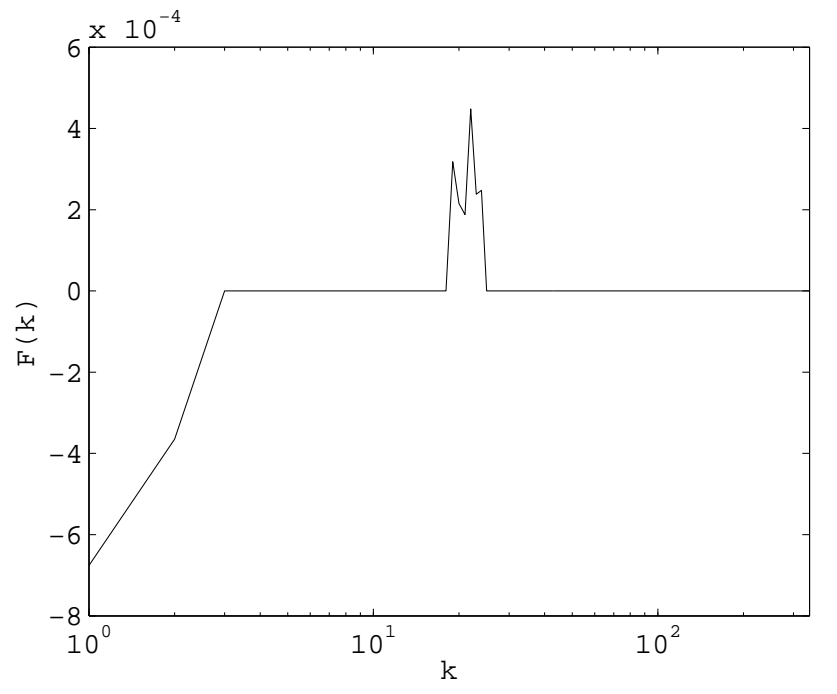

Figure 13: Contribution of the forcing to the energy spectrum at $t=\frac{19}{20} T$.

flow under linear forcing are similar to the case where the conventional band-limited forcing active over largest scales is used.

In general, full-band forcings are of interest because they are physically more realistic than band-limited forcings. Although there is no reason to believe that the precise optimal forcing found here may be observed in nature, it suggests that employing more realistic forcings (rather than the random-phase band-limited forcing) in the studies of two-dimensional turbulence may significantly change the dynamics of the flow, including those of the dual cascade. In addition, full-band forcings are much easier to implement in numerical simulations, which do not benefit from the scale localization of spectral methods.

We also found that the optimal forcing automatically creates a sink of energy at largest scales. It has already been proved (Constantin et al. 1994; Tran \& Shepherd 2002; Tran \& Bowman 2003) in the case of monoscale forcing that such an infrared sink is a necessary condition in order to obtain the dual cascades and KLB scaling laws. We have observed a similar sink even when the forcing is full-band. Our result suggests the possibility to generalize the existing proofs to more general types of forcing.

In the case of band-limited forcing, the optimal control method still finds a forcing which results in the KLB scaling laws. However, the -3 range of the spectrum extends over only a quarter of a decade of wavenumbers. It is possible, however, to extend this scaling range by increasing the resolution (results not presented here).

Comparison of the energy spectra resulting from our optimal forcing and from the conventional random-phase band-limited forcing (figure 9) suggests that the details of the space-time structure of the forcing can crucially alter the statistical properties of the flow. For example figure 11 shows that the optimal forcing is particularly aligned in the favour of enstrophy injection. Replacing the phase-structure of the optimal forcing with a random variable (and preserving other properties of it) leads to a much steeper spectrum of the enstrophy cascade (i.e. $E \sim k^{-4}$ ). This shows that the conventional forcing (which is usually random in phase) has determining effects on the scaling properties of the enstrophy cascade. 
On the other hand, small perturbations in the band-limited optimal forcing leads to a much steeper fall-off of the energy spectrum (close to $k^{-4}$ ). Moreover, we have not succeeded in constructing a simple model of the forcing based on the observed space-time properties of our optimal forcing. These observations imply that solutions of optimization problem (2.5) are quite sensitive to perturbations which is to be expected given the illposed character of the problem. This suggests that the optimal forcing belongings to a sparse set in the space of square-integrable band-limited functions. Therefore, it is quite unlikely to be physically realizable. This implies that reproducing the co-existing dual cascades which follow the KLB scaling laws is unlikely when a band-limited forcing is used with a moderate Reynolds number.

Finally, we emphasize that the method of controlling the energy spectrum of the flow introduced here can be used for other problems in fundamental turbulence research. For example, by making a particular choice of the weight function $w(t, k)$ in equation $(2.4)$ one can control the rate at which an initially localized energy spectrum spreads over all scales. This can provide a different perspective to some problems such as transition to turbulence and drag (or lift) control in a flow over a rigid body which have already been studied through other approaches. Moreover, Gioia \& Chakraborty (2006) showed that the wall friction in a turbulent flow depends significantly on the energy spectrum of the flow. Therefore by controlling the energy spectrum one can also control the turbulent friction in pipe flow. The method could also be used in some benchmark studies in geophysical fluid dynamics. For instance, in a separate attempt (not published) we have produced the Gage-Nastrom spectrum (Gage \& Nastrom 1985) of mesoscale atmospheric flow in a forced two-dimensional Navier-Stokes flow. Furthermore, our method can be easily modified to control the energy spectrum of the geophysical fluid dynamics models such as the quasi-geostrophic and surface quasi-geostrophic equations.

In summary, we have used an optimal control theory approach to find a forcing that produces the simultaneous dual scaling ranges predicted by KLB. Previous studies (Boffetta \& Musacchio 2010; Bracco \& McWilliams 2010) have presented strong evidence in support of KLB scaling in the limit of very large Reynolds numbers. In the present study, the simultaneous dual cascade with the KLB scaling has been observed for the first time at a relatively low Reynolds number by employing a non-conventional forcing. However, the high sensitivity of the energy spectrum to this forcing suggests that it is unlikely to be observed in nature or in laboratory experiments.

\section{REFERENCES}

Basdevant, C., Legras, B. \& Sadourny, R. 1981 A study of barotropic model flows: Intermittency, waves and predictability. J. Atmos. Sci. 38, 2305-2326.

Batchelor, G. K. 1969 Computation of the energy spectrum in homogeneous two-dimensional turbulence. Phys. Fluids Suppl. II, 233-239.

Bewley, T. R. 2001 Flow control: new challanges for a renaissance. Prog. Aerosp. Sci. 37, $21-58$.

Boffetta, G. 2007 Energy and enstrophy fluxes in the double cascade of two-dimensional turbulence. J. Fluid Mech. 589, 253-260.

Boffetta, G., Celani, A., Musacchio, S. \& Vergassola, M. 2002 Intermittency in twodimensional Ekman-Navier-Stokes turbulence. Phys. Rev. E 66, 026304.

Boffetta, G. \& Musacchio, S. 2010 Evidence for the double cascade scenario in twodimensional turbulence. Phys. Rev. E 82, 016307.

Borue, V. 1993 Spectral exponents of enstrophy cascade in stationary two-dimensional homogeneous turbulence. Phys. Rev. Lett. 71, 3967.

Bracco, A. \& McWilliams, J. C. 2010 Reynolds-number dependency in homogeneous, stationary two-dimensional turbulence. J. Fluid Mech. 646, 517-526. 
Bruneau, C. H. \& Kellay, H. 2005 Experiments and direct numerical simulations of twodimensional turbulence. Phys. Rev. E 71, 046305.

Chen, S., Ecke, R. E., Eyink, G. L., Wang, X. \& Xiao, Z. 2003 Physical mechanism of the two-dimensional enstrophy cascade. Phys. Rev. Lett. 91, 214501.

Clercx, H. J. H. \& van Heisst, G. J. F. 2009 Two-dimensional Navier-Stokes turbulence in bounded domains. Appl. Mech. Rev. 62, 020802.

Constantin, P., Foias, C. \& Manley, O. 1994 Effects of the forcing function spectrum on energy spectrum in 2-D turbulence. Phys. Fluids 6, 427-429.

Edwards, W. S., Tuckerman, L. S., Friesner, R. A. \& Sorensen, D. C. 1994 Krylov methods for the incompressible Navier-Stokes equations. J. Comp. Phys. 110, 82-102.

FJøRTOFт, R. 1953 On the changes in the spectral distribution of kinetic energy for two dimensional, nondivergent flow. Tellus 5, 225-230.

FRISCH, U. 1995 Turbulence: the legacy of A.N. Kolmogorov. Cambridge University Press.

Frisch, U. \& Sulem, P. L. 1984 Numerical simulation of inverse cascade in two-dimensional turbulence. Phys. Fluids 27, 1921-1923.

Gage, K. S. \& NASTrom, G. D. 1985 On the spectrum of the atmospheric velocity fluctuations seen by MST/ST radar and their interpretation. Radio Sci. 20, 1339-1374.

Gioia, G. \& Chakraborty, P. 2006 Turbulent friction in rough pipes and the energy spectrum of the phenomenological theory. Phys. Rev. Lett. 96, 044502.

GkioulekAs, E. \& Tung, K. K. 2007 A new proof on net upscale energy cascade in twodimensional and quasi-geostrophic turbulence. J. Fluid Mech. 576, 173-189.

Gunzburger, M. D. 2003 Perspectives in flow control and optimization. SIAM.

Kolmogorov, A. K. 1941 The local structure of turbulence in incompressible viscous fluid for very large Reynolds numbers. Dokl. Akad. Nauk. 30, 301-305.

Kraichnan, R. H. 1967 Inertial ranges in two-dimensional turbulence. Phys. Fluids 10, 14171423.

Kraichnan, R. H. 1971 Inertial-ranges transfer in two- and three-dimensional turbulence. J. Fluid Mech. 47, 525-535.

Lebedev, L. P. \& Vorovich, I. I. 2002 Functional Analysis in Mechanics. Springer.

Leith, C. E. 1968 Diffusion approximation for two-dimensional turbulence. Phys. Fluids 11, 671-673.

Lilly, D. D. 1969 Numerical simulation of two-dimensional turbulence. Phys. Fluids Suppl. II, 240-249.

LindBorG, E. 1999 Can the atmospheric kinetic energy spectrum be explained by twodimensional turbulence? J. Fluid Mech. 388, 259-288.

Lions, J. L. 1969 Contrôle optimal des systèmes gouvernés par des equations aux dérivées partialles. Dunod, Paris, (English translation, Springer-Verlag, New York).

Lundgren, T. S. 2003 Linearly forced isotropic turbulence. Tech. Rep.. Center for Turbulence Research.

Maltrud, M. E. \& Vallis, G. K. 1993 Energy and enstrophy transfer in numerical simulations of two-dimensional turbulence. Phys. Fluids A 5, 1760-1775.

Marteau, D., Cardoso, O. \& Tabeling, P. 1995 Equilibrium states of two-dimensional turbulence: An experimental study. Phys. Rev. E 51, 5124-5127.

Merilees, P. E. \& WARN, H. 1975 On energy and enstrophy exchanges in two-dimensional non-divergent flow. J. Fluid Mech. 69, 625-630.

Nocedal, J. \& Wright, S. J. 2000 Numerical Optimization. Springer.

Pasquero, C. \& FAlkovich, G. 2002 Stationary spectrum of vorticity cascade in twodimensional turbulence. Phys. Rev. E 65, 056305.

Protas, B., Bewley, T. R. \& Hagen, G. 2004 A comprehensive framework for the regularization of adjoint analysis in multiscale PDE systems. J. Comp. Phys. 195, 49-89.

Rosales, C. \& Meneveau, C. 2005 Linear forcing in numerical simulations of isotropic turbulence: Physical space implementation and convergence studies. Phys. Fluids 17, 095106.

Schorghofer, N. 2000 Energy spectra of steady two-dimensional turbulent flows. Phys. Rev. E 61, 6575-6577.

Schulze, J. C., Schmid, P. J. \& Sesterhenn, J. L. 2009 Exponential time integration using Krylov subspaces. Int. J. Numer. Meth. Fluids 60, 591-609. 
Scott, R. K. 2007 Non-robustness of the two-dimensional turbulent inverse cascade. Phys. Rev. E 75, 046301.

Sмiтн, L. M. \& Yакнот, V. 1993 Bose condensation and small-scale structure generation in a random force driven 2d turbulence. Phys. Rev. Lett. 71, 352-355.

Sommeria, J. 1986 Experimental study of the two-dimensional inverse energy cascade in a square box. J. Fluid Mech. 170, 139-168.

Tran, C. V. \& Bowman, J. C. 2003 On the dual cascade in two dimensional turbulence. Physica D 176, 242-255.

Tran, C. V. \& Dritschel, D. G. 2006 Vanishing enstrophy dissipation in two-dimensional Navier-Stokes turbulence in the inviscid limit. J. Fluid Mech. 559, 107-116.

Tran, C. V., Dritschel, D. G. \& Scott, R. K. 2007 Revisiting batchelor's theory of twodimensional turbulence. J. Fluid Mech. 591, 379-391.

Tran, C. V. \& Shepherd, T. G. 2002 Constraints on the spectral distribution of energy and enstrophy dissipation in forced two-dimensional turbulence. Physica D 165, 199-212.

Tsang, Y.-K., Ott, E., Antonsen, T. M. \& Guzdar, P. N. 2005 Intermittency in twodimensional turbulence with drag. Phys. Rev. E 71, 066313.

Tsang, Y.-K. \& Young, W. R. 2009 Force-dissipative two-dimensional turbulence: A scaling regime controlled by drag. Phys. Rev. E 79, 045308(R).

Welch, W. T. \& Tung, K. K. 1998 Nonlinear baroclinic adjustment and wavenumber selection in a simple case. J. Atmos. Sci. 55, 1285-1302. 